\title{
Some THEMIS-MTR observations of the second solar spectrum (2000 campaign) ${ }^{\star}$
}

\author{
V. Bommier ${ }^{1}$ and G. Molodij ${ }^{2}$ \\ 1 Laboratoire "Atomes et Molécules en Astrophysique", CNRS UMR 8588 - DAMAp, Observatoire de Paris, \\ Section de Meudon, 92195 Meudon, France \\ 2 THEMIS S.L., c/o Instituto de Astrofísica de Canarias, 38200 La Laguna, Tenerife, Canary Islands, Spain ${ }^{\star \star}$
}

Received 9 February 2001 / Accepted 18 October 2001

\begin{abstract}
We report spectropolarimetric observations with the THEMIS telescope multi-lines operating mode (MTR) during the 2000 observational period from August 27th to September 1st. We measured the resonance polarization at the limb of a series of lines: Sr I $460.7 \mathrm{~nm}$, Na I D $589.6 \mathrm{~nm}$ and $\mathrm{D}_{2} 589.0 \mathrm{~nm}$, Ba II D $493.4 \mathrm{~nm}$ and $\mathrm{D}_{2} 455.4 \mathrm{~nm}, \mathrm{C}_{\text {I }} 493.2 \mathrm{~nm}$. The data analysis method is mainly described in Bommier \& Rayrole (2002), and has been completed by using the beam exchange facility as available in 2000 THEMIS, i.e., in a single Stokes parameter. A so-called "generalized beam exchange" technique has been settled on, for the full Stokes vector measurement under this limitation. The observations have been devoted to the measurement of the scattering polarization which is a linear polarization observed near the limb of the Quiet Sun, eventually modified by a weak magnetic field (the so-called Hanle effect). The entrance slit of the spectrograph has been oriented parallel to the tangential direction of the solar limb, and data have been averaged in time and along the spatial direction of the slit in order to increase the polarimetric resolution. Two different cameras have been used to record simultaneously the two polarization states exiting the beam-splitter. The results of our polarization measurements are in good agreement with those given in the second spectrum solar atlas of Gandorfer (2000), based on 1999-2000 observations. Nevertheless, with regard to a quantification of the polarization signal, we found that the signal is systematically smaller than previous results obtained during the 1994-96 observational period and was also observed as decreasing during the 1998 observational period, as if a 11-year cyclic variation of the limb polarization occured. This signal variability obviously requires further observational and interpretative investigations. We noticed other differences to previous results, in particular, the linear polarization shape of the $\mathrm{Na}$ I $\mathrm{D}_{1}$ line that also requires further observational investigation.
\end{abstract}

Key words. methods: data analysis - methods: observational - techniques: polarimetric - techniques: spectroscopic - Sun: atmosphere - Sun: magnetic fields

\section{Introduction}

The limb and center-to-limb polarization of the resonance Sr I $460.7 \mathrm{~nm}$ line has already been measured in quiet regions of the Sun (Stenflo et al. 1980, 1983, 1997). The aim of the present work is first to measure this line polarization (in the quiet solar regions) and compare the results with previous measurements, in order to test the possibility of achieving such measurements with the French-Italian

Send offprint requests to: V. Bommier,

e-mail: V.Bommier@obspm.fr

* Based on observations made with THEMIS operated on the island of Tenerife by CNRS-CNR in the Spanish Observatorio del Teide of the Instituto de Astrofísica de Canarias.

** Permanent address: Laboratoire "Physique du Soleil et de l'Héliosphère", CNRS UMR 8645 - DASOP, Observatoire de Paris, Section de Meudon, 92195 Meudon, France. solar THEMIS telescope (THEMIS is a French acronym which stands for "Télescope Héliographique pour l'Étude du Magnétisme et des Instabilités Solaires") operating for its third campaign. The measurements of the most linearly polarized lines of the solar spectrum observed near the limb in quiet regions (Stenflo et al. 1983) require a very high polarimetric sensitivity. The linear polarization signals produced by the atomic polarization are weak and are not "a priori" included in the THEMIS objectives. Moreover, all the most linearly polarized lines such as the Sr I $460.7 \mathrm{~nm}$ one, fall in the $400-500 \mathrm{~nm}$ spectral region away from the nominal wavelength bandwidth for which THEMIS has been optimized, namely 500-900 nm. For these reasons, such present test observations are highly desirable.

Secondly, the interpretation of the limb polarization of the Sr I $460.7 \mathrm{~nm}$ line is an interesting and, in our opinion, 
a remaining open question. The $460.7 \mathrm{~nm}$ line is the resonance line of Sr $\mathrm{s}: 5 \mathrm{~s}^{2}{ }^{1} \mathrm{~S}_{0} \rightarrow 5 \mathrm{~s} 5 \mathrm{p}^{1} \mathrm{P}_{1}$ (the Landé factor for the upper level $J^{\prime}=1$ is $g_{J^{\prime}}=1$ ). When observing near the solar limb in quiet solar regions, the line is linearly polarized, due to the scattering of the underlying anisotropic radiation. This anisotropy results from the radiative transfer near the surface of the layer where the line is formed.

The performed modeling of the line formation, using non-LTE modeling gives polarization signals higher than observed ones. The difference between the observed and computed polarizations is ascribed to be the depolarizing effect of a weak magnetic field (Hanle effect). However, the observed polarization direction remains parallel to the tangential direction of the solar limb, which is the polarization direction in the absence of a magnetic field and is in contradiction to the effect of a homogeneous weak magnetic field acting to rotate the polarization direction with respect to the limb direction. To interpret the observations (i.e., the absence of rotation of the polarization direction), one assumes that the magnetic field has an unresolved turbulent structure. Non-LTE modeling taking into account the effect of such a turbulent magnetic field leads to the determination of the turbulent field strength (FaurobertScholl 1993; Faurobert-Scholl et al. 1995). Within this modeling, the observed center-to-limb variation can be recovered only if one assumes the presence of a field strength that rapidly varies along the depth. The value of the average field strength expected is around 10-20 Gauss.

On the one hand, interpretation of observations of the linear polarization of the $\mathrm{Na}_{\mathrm{I}} \mathrm{D}_{1}$ and $\mathrm{D}_{2}$ lines (Stenflo \& Keller 1997; Stenflo et al. 2000a), in terms of lower level atomic polarization effect, rules out the existence of a turbulent magnetic field strength higher than $10 \mathrm{mG}$ (Landi Degl'Innocenti, 1998) in the layer where the $\mathrm{Na}_{\mathrm{I}} \mathrm{D}_{1}$ and $\mathrm{D}_{2}$ lines are formed (which is $\sim 200 \mathrm{~km}$ above the layer where the SrI $460.7 \mathrm{~nm}$ line is formed). On the other hand, the modeling of the turbulent magnetic field effect of Faurobert-Scholl (1993) and Faurobert-Scholl et al. (1995), uses angle-averaged frequency redistribution functions. Previous works of Faurobert (1987, see Fig. 1, and 1988, see Fig. 1) compare the effect of angle-dependent and angle-averaged redistribution functions without a magnetic field and directly implies that angular effects play an important role in the line-core center and have to be taken into account in scattering polarization modeling. Nevertheless, averaging the angular dependence of the frequency redistribution function assumes a complete velocity redistribution during the scattering process. This phenomenon is not compatible with the existence of nonzero atomic polarization (which is responsible for the scattering polarization), and implies high collisional rates to assure the complete velocity redistribution (with respect to the radiative rates). In this case, the atomic polarization would not survive (as has been discussed in Landi Degl'Innocenti 1995).

It can be concluded that spectropolarimetric observations have to be carried out, as well as new non-LTE
Table 1. Spectral lines observed in the present work. $\lambda$ : wavelength in nm. $W$ : equivalent width in pm.

\begin{tabular}{cllrr}
\hline \hline $\begin{array}{c}\lambda \\
\mathrm{nm}\end{array}$ & Element & line & $\begin{array}{r}\text { multiplet } \\
\#\end{array}$ & $\begin{array}{r}W \\
\mathrm{pm}\end{array}$ \\
\hline \hline 455.4035 & Ba II & $\mathrm{D}_{2}$ & 1 & 16.71 \\
455.4991 & Cr I & & 44 & 3.92 \\
\hline 460.7333 & Sr I & & 2 & 3.59 \\
460.7651 & Fe I & & 554 & 7.51 \\
\hline 493.2072 & C I & & 13 & 3.70 \\
493.3194 & Fe I & & 1070 & 3.90 \\
493.3343 & Fe I & & 1065 & 9.72 \\
493.3877 & Fe I & & 968 & 4.09 \\
493.4084 & Ba II & $\mathrm{D}_{1}$ & 1 & 13.72 \\
\hline 588.9966 & Na I & $\mathrm{D}_{2}$ & 1 & 70.67 \\
589.2879 & Ni I & & 68 & 6.60 \\
589.5932 & Na I & $\mathrm{D}_{1}$ & 1 & 53.64 \\
\hline \hline
\end{tabular}

calculations taking into account angle-dependent redistribution effects. Notice that a polarimetric sensitivity of 2 $4 \times 10^{-4}$ has been reached during the first campaign observations of THEMIS in the continuum around the Fe I $557.6 \mathrm{~nm}$ line (Bommier \& Rayrole 2002) when the spectra are averaged along the entrance slit of the spectrograph (corresponding to $\sim 250$ camera pixels, i.e. $\sim 2$ arcmin on the solar disk) and when $\sim 150$ frames are averaged. So, the potential interest of THEMIS for the observation of weak polarization signals is expected in order to measure the linear polarization of the Sr I $460.7 \mathrm{~nm}$ line with a linecore amplitude around $1.35-1.75 \%$ at 5 arcsec inside the solar limb, as already found by Stenflo et al. (1997).

During the 2000 campaign period, one of our goals was to investigate the capability of THEMIS to accurately measure polarization in the $10^{-3}$ amplitude range. In this respect, we observed also the spectral lines listed in Table 1, whose polarization has previously been observed in this domain of sensitivity (Stenflo \& Keller 1997; Stenflo et al. 2000a, 2000b).

Two lines of the mentioned list are $\mathrm{D}_{2}$ lines, with upper level $J^{\prime}=3 / 2$ and lower level $J=1 / 2$, whereas two other ones are $\mathrm{D}_{1}$ lines, with upper level $J^{\prime}=1 / 2$ and lower level $J=1 / 2$. The $\mathrm{D}_{2}$ lines may be globally linearly polarized when neglecting an eventual hyperfine structure while the $\mathrm{D}_{1}$ lines may not, (i.e., in that case we ignore the redistribution details when integrated over frequencies). Notice that the sodium and baryum nuclei have a non-zero nuclear spin and then, a hyperfine structure which acts always as a depolarizing mechanism. So far, the $\mathrm{D}_{1}$ lines should remain unpolarized when integrating over frequencies. This is the case for the theoretical modeling of the $\mathrm{NaID}_{1}$ polarization spectrum performed by Landi Degl'Innocenti (1998), for which coherent scattering as well as quantum intereferences with the neighbouring $\mathrm{Na} \mathrm{I} \mathrm{D}_{2}$ line are taken into account. However, the previous 
Table 2. Sequence of 2000 THEMIS polarization analyzer QWP (quarter-wave plates) positions, used in the present work. The orientations of the two quarter-wave plates QWP1 and QWP2 are referred with respect to the beam-splitter axis, which is also the reference axis for the Stokes parameters definition, in this table (see text). QWP1 is the first plate encountered by the incoming radiation.

\begin{tabular}{ccrrc}
\hline $\begin{array}{c}\text { Pos. } \\
\#\end{array}$ & $\begin{array}{c}\text { Pos. } \\
\text { name }\end{array}$ & QWP1 & QWP2 & $\begin{array}{c}\text { Output } \\
\text { Signal }\end{array}$ \\
\hline 1 & $Q$ & $0^{\circ}$ & $0^{\circ}$ & $I \pm Q$ \\
2 & $U$ & $0^{\circ}$ & $45^{\circ}$ & $I \pm U$ \\
3 & $I Q$ & $45^{\circ}$ & $45^{\circ}$ & $I \mp Q=I \pm I Q$ \\
4 & $V$ & $45^{\circ}$ & $0^{\circ}$ & $I \mp V$ \\
\hline
\end{tabular}

observations of the two $\mathrm{D}_{1}$ lines listed in Table 1 (Stenflo \& Keller 1997; Stenflo et al. 2000a; 2000b) show positive peaks in their linear polarization (i.e., non-zero linear polarization when integrated over frequencies), that are unexpected from a theoretical point-of-view. In this respect, the measurement of these linear line polarizations is an interesting goal.

Section 2 presents the observational technique. We paid particular attention to the treatment of the fringe patterns produced by the polarization analyzer. The data analysis method, which is based on Bommier \& Rayrole (2002), is described in Sect. 3. One of the aims of the present paper is also to describe a generalization of the beam exchange technique for polarimetry to the situation encountered during the 2000 campaign of THEMIS. The powerful beam exchange technique has been already successfully applied to stellar astronomy (see in particular Donati et al. 1990) and solar astronomy (see in particular Bianda et al. 1998), and could be performed only for a single Stokes parameter with the polarimeter available during the 2000 observational period of THEMIS. We generalize the method to retrieve all three polarization Stokes parameters with quite the same level of accuracy, by using the beam exchange in a single Stokes parameter. This method is described in Appendix A. The results of our observations are given and discussed in Sect. 4.

\section{Observational procedure with THEMIS, MTR mode}

We have carried out the observations during the 2000 campaign from August 27th to September 1st with the THEMIS multi-line spectropolarimetry mode (MTR). We briefly describe below this mode operation.

THEMIS is a Richtey-Chrétien telescope supported by an azimuthal mount. It is therefore pointing directly at the Sun. The telescope would be evacuated, but is actually filled with helium in light excessive pressure and closed by two windows. The polarization analyzer is located at the telescope's prime focus, before any oblique reflection in the optical path. Hence, THEMIS is a "polarization free" telescope. The polarization analyzer, located at focus F1, is divided in 3 parts: 2 achromatic quarter-wave plates and a polarizing beam splitter. The beam splitter axes are oriented at 45 degrees to the spectrograph entrance slit. The axes of the two quater-wave plates are oriented so that the 3 Stokes parameters combinations $I+V$ and $I-V$, $I+U$ and $I-U, I+Q$ and $I-Q$ are obtained successively at the output of the analyzer. The subsequent optics are designed for the transfer of the two useful beams coming out of the analyzer along close optical paths. The transfer optics produces an enlarged image on the entrance slit of the spectrograph (secondary focus F2, equivalent focal length of 57 meters, F/63). In F2, the polarization analyzer produces 2 images of the window placed in front of the polarization analyzer (focus F1). The length of the images is 2 arcmin and the width can be adjusted from 2 to 12 arcsec depending on the Fried parameter $r_{0}$. The two useful beams are adjusted along the spectrograph slit by means of an optical prism device so that the two spectra, for the two beams transmitted by the polarization analyzer, will be completely separated at the focus (SP2) of the echelle spectrograph. The spectrograph hangs vertically from the same part as the azimuthal mount of the telescope. Both beams enter the long predisperser and pass through the collimator which can compensate astigmatism aberrations. Three different gratings can be exchanged automatically in the predisperser, (one of them is an echelle grating). In the basic configuration, masks can be put in the plane of the intermediate focus (SP1) in order to select a number of spectral lines. The large camera mirror of the echelle spectrograph produces high dispersion spectra simultaneously for all the spectral ranges selected in the predisperser focus (SP1) of the two beams transmitted by the analyzer of polarization. The last transfer optics (to adjust the size of the pixel) and the detectors are placed at the focus (SP2) of this spectrograph. The basic configuration includes twenty CCD cameras (286x 384 pixels), which are necessary to record simultaneously 10 line profiles in two Stokes parameters along 382 solar points. The recorded images are then two-dimensional images: the horizontal dimension is the wavelength while the vertical dimension is the solar coordinate along the entrance slit. Another camera is used to record the fieldof-view window in front of the polarization analyzer (focus F1).

The polarimeter consists of two identical achromatic crystalline quarter-wave plates (or QWPs) followed by a calcite beam-splitter. The QWPs are achromatic over a 400-700 nm spectral range; hence the phase of each of the QWP is $90 \pm 4^{\circ}$ in that range. Each QWP can be oriented at 3 different discrete angular positions over $45^{\circ}$ range, $22.5^{\circ}$ stepwise. Table 2 lists the sequence used during the observation. We denote as $I X$ the "inverse" of the Stokes parameter $X$ when exchanging the two beams (see Table 2). Notice that only beam exchange of the $Q$ Stokes parameter was avalaible in the 2000 THEMIS configuration. 
Table 3. General information about the present observations. Column a: reference number of the observation in the present work. Column b-c: date and time (UT) of the observation. Column d: central wavelength of the spectral window. Column e: magnifying factor of the camera optics. Columns $\mathrm{f}-\mathrm{g}$ : spectrograph passing band (PB), in pm and in terms of camera pixel respectively. Column h: number of recorded images, for each analyzer position or Stokes parameter (the second number is the images number in an individual slice of images, the first number is the total number of images slices). Column i: integration time for one image, in seconds. Column j: limb distance for the spectrograph slit, in arcsec. Column k: cosine of the heliocentric angle.

\begin{tabular}{ccccccccccc}
\hline \hline $\mathrm{a}$ & $\mathrm{b}$ & $\mathrm{c}$ & $\mathrm{d}$ & $\mathrm{e}$ & $\mathrm{f}$ & $\mathrm{g}$ & $\mathrm{h}$ & $\mathrm{i}$ & $\mathrm{j}$ & $\mathrm{k}$ \\
\hline $\begin{array}{c}\text { Obs. } \\
\#\end{array}$ & date & time & $\lambda$ & $\gamma$ & $\mathrm{PB}$ & $\mathrm{PB}$ & $N_{\mathrm{im}}$ & $t_{\text {int }}$ & $d_{\mathrm{limb}}$ & $\mu$ \\
\hline \hline$\# 1$ & $00 / 08 / 27$ & $08 \mathrm{~h} 20-09 \mathrm{~h} 19$ & 460.7 & 0.44 & 1.97 & 2.32 & $2 \times 10$ & 15 & $5.9 \pm 0.4$ & $0.110 \pm 0.004$ \\
$\# 2$ & $00 / 08 / 27$ & $08 \mathrm{~h} 47-09 \mathrm{~h} 54$ & 460.7 & 0.44 & 1.97 & 2.32 & $2 \times 10$ & 15 & $7.0 \pm 0.2$ & $0.121 \pm 0.002$ \\
$\# 3$ & $00 / 08 / 28$ & $08 \mathrm{~h} 14-10 \mathrm{~h} 09$ & 460.7 & 0.44 & 1.97 & 2.32 & $3 \times 10$ & 17 & $3.4 \pm 0.4$ & $0.084 \pm 0.005$ \\
\hline$\# 4$ & $00 / 08 / 29$ & $07 \mathrm{~h} 52-08 \mathrm{~h} 33$ & 589.6 & 0.23 & 2.59 & 1.22 & $2 \times 25$ & 1.5 & $4.3 \pm 0.5$ & $0.094 \pm 0.006$ \\
$\# 5$ & $00 / 08 / 29$ & $08 \mathrm{~h} 51-09 \mathrm{~h} 28$ & 589.0 & 0.23 & 2.60 & 1.21 & $2 \times 25$ & 1.0 & $4.0 \pm 0.5$ & $0.092 \pm 0.006$ \\
$\# 6$ & $00 / 08 / 29$ & $09 \mathrm{~h} 28-10 \mathrm{~h} 03$ & 589.3 & 0.23 & 2.59 & 1.21 & $2 \times 25$ & 1.0 & $4.1 \pm 0.5$ & $0.093 \pm 0.006$ \\
\hline$\# 7$ & $00 / 08 / 29$ & $15 \mathrm{~h} 15-15 \mathrm{~h} 45$ & 589.6 & 0.23 & 2.59 & 1.22 & $2 \times 25$ & 0.9 & $3.6 \pm 0.5$ & $0.086 \pm 0.005$ \\
$\# 8$ & $00 / 08 / 29$ & $15 \mathrm{~h} 45-16 \mathrm{~h} 13$ & 589.0 & 0.23 & 2.60 & 1.21 & $2 \times 25$ & 0.9 & $4.1 \pm 0.6$ & $0.093 \pm 0.006$ \\
$\# 9$ & $00 / 08 / 29$ & $16 \mathrm{~h} 13-16 \mathrm{~h} 36$ & 589.3 & 0.23 & 2.59 & 1.21 & $2 \times 25$ & 0.9 & $4.0 \pm 0.7$ & $0.091 \pm 0.008$ \\
\hline$\# 10$ & $00 / 08 / 30$ & $15 \mathrm{~h} 30-16 \mathrm{~h} 14$ & 589.0 & 0.44 & 2.61 & 2.31 & $2 \times 20$ & 2.5 & $4.1 \pm 0.4$ & $0.093 \pm 0.005$ \\
$\# 11$ & $00 / 08 / 30$ & $16 \mathrm{~h} 14-17 \mathrm{~h} 09$ & 589.6 & 0.44 & 2.60 & 2.32 & $3 \times 20$ & 2.5 & $3.8 \pm 0.6$ & $0.089 \pm 0.007$ \\
\hline$\# 12$ & $00 / 09 / 01$ & $08 \mathrm{~h} 33-09 \mathrm{~h} 21$ & 493.4 & 0.44 & 2.05 & 2.30 & $2 \times 15$ & 6 & $4.1 \pm 0.4$ & $0.092 \pm 0.005$ \\
$\# 13$ & $00 / 09 / 01$ & $09 \mathrm{~h} 21-10 \mathrm{~h} 06$ & 493.2 & 0.44 & 2.06 & 2.31 & $2 \times 15$ & 6 & $4.3 \pm 0.5$ & $0.095 \pm 0.006$ \\
\hline$\# 14$ & $00 / 09 / 01$ & $16 \mathrm{~h} 09-17 \mathrm{~h} 15$ & 455.4 & 0.23 & 1.86 & 1.22 & $14+10$ & 14 & $4.8 \pm 1.0$ & $0.100 \pm 0.010$ \\
\hline \hline
\end{tabular}

In the present section devoted to instrumental features, the reference system for the Stokes parameters definition is the one of the beam-splitter, where the $O X$ axis (the "positive $Q$ direction") is one of the beam-splitter axes, and is oriented $45^{\circ}$ from the spectrograph entrance slit.

We have denoted each combination of QWP positions by the name of the Stokes parameter which is measured with this position. The positions are listed in Table 2 in the order of our sequence, which is then

$(Q, U, I Q, V)$

This sequence is repeated $N_{\text {im }}$ times, $N_{\text {im }}$ being the number of images recorded in each Stokes parameter (the total number of recorded images being then $4 \times N_{\text {im }}$ ).

The entrance slit of the spectrograph has been oriented parallel to the tangential direction of the solar limb, and data have been averaged in time and along the spatial direction of the slit, in order to increase the polarimetric resolution. The photon noise on the final averaged spectrum $\sigma$ phot can be determined by

$\sigma_{\text {phot }}=\frac{\sqrt{N}}{N \sqrt{N_{\text {im }}} \sqrt{N_{\text {row }}}}$,

where $N$ is the number of photons

$N=I_{\mathrm{ADU}} \times 235$, derived from the recorded ADU level $I_{\mathrm{ADU}}$ taken in a flat continuum region, and from the number of photons per detected electron which is $\sim 235$ on the THEMIS cameras, and where $N_{\text {row }}$ is the number of used rows of the camera (maximum 286 for the 2000 THEMIS configuration). In the present observations, the level of photon noise value is around few $10^{-5}$ as indicated in Table 4 . Notice also that the fluctuations of the derived polarization spectrum, also given in Table 4, are close to the photon noise limit. These fluctuations correspond to the calculation of the standard deviation of the flat profile obtained when subtracting the result of the wavelet filtering operation of the polarization profile described in Bommier \& Rayrole (2002).

As done during the 1998 observational period (Bommier \& Rayrole 2002), the flat-field record has been taken on the Sun itself by moving rapidly the solar disk on the entrance slit, along a random ellipse. Ellipse axes were smaller in the 2000 observations than in the 1998 ones in order to reduce the line position variations due to the solar rotation and Doppler effect during the temporal sequence of the flat-field images. As a consequence, the line profile is even less visible in the flat-field matrix in the 2000 data than in the 1998 ones, increasing the quality of the flat-field matrix (as shown in Fig. 3 of Bommier \& Rayrole 2002).

Comparated to the 1999 (unpublished) data, we reduced the difference between limb and flat-field fringe 
Table 4. Column 1: photon noise in the present observations (average between the two cameras). Columns m-o: polarization fluctuations along the spectra obtained in the present observations, in $Q / I, U / I$ and $V / I$ respectively. $Q / I$ and $V / I$ are obtained by using the generalized beam exchange technique introduced in the present paper, whereas $U / I$ is obtained by using the usual beam exchange technique. In this table, the reference axis for the Stokes parameters definition is the solar limb.

\begin{tabular}{cccccc}
\hline \hline $\mathrm{a}$ & $\mathrm{d}$ & $\mathrm{l}$ & $\mathrm{m}$ & $\mathrm{n}$ & $\mathrm{o}$ \\
\hline Obs. & $\lambda$ & $\sigma_{\mathrm{phot}}$ & $\sigma_{Q}$ & $\sigma_{U}$ & $\sigma_{V}$ \\
$\#$ & $\mathrm{~nm}$ & $\times 10^{-4}$ & $\times 10^{-4}$ & $\times 10^{-4}$ & $\times 10^{-4}$ \\
\hline \hline$\# 1$ & 460.7 & 0.55 & 1.6 & 0.9 & 1.9 \\
$\# 2$ & 460.7 & 0.51 & 1.6 & 0.8 & 1.3 \\
$\# 3$ & 460.7 & 0.49 & 1.4 & 1.0 & 1.4 \\
\hline$\# 4$ & 589.6 & 0.18 & 0.8 & 0.3 & 0.5 \\
$\# 5$ & 589.0 & 0.21 & 0.7 & 0.3 & 0.5 \\
$\# 6$ & 589.3 & 0.19 & 0.6 & 0.3 & 0.4 \\
\hline$\# 7$ & 589.6 & 0.19 & 0.4 & 0.2 & 0.3 \\
$\# 8$ & 589.0 & 0.20 & 0.5 & 0.2 & 0.5 \\
$\# 9$ & 589.3 & 0.19 & 0.4 & 0.2 & 0.5 \\
\hline$\# 10$ & 589.0 & 0.23 & 0.6 & 0.2 & 0.7 \\
$\# 11$ & 589.6 & 0.18 & 0.5 & 0.2 & 0.6 \\
\hline$\# 12$ & 493.4 & 0.32 & 0.8 & 0.4 & 0.7 \\
$\# 13$ & 493.2 & 0.30 & 0.7 & 0.5 & 0.6 \\
\hline$\# 14$ & 455.4 & 0.37 & 2.3 & 1.6 & 2.6 \\
\hline \hline
\end{tabular}

patterns formed in the polarization analyzer applying the following observational strategy:

- we interleave limb observations and flat-field records, giving each a $\sim 10 \mathrm{mn}$ time duration. The number of images taken in each Stokes parameter, $N_{\mathrm{im}}$, is then given in column $\mathrm{h}$ of Table 3 by the number of such $\sim 10 \mathrm{mn}$ time sequences, times the number of images taken during one $\sim 10 \mathrm{mn}$ sequence;

- we correct the instrumental drift at each record change (every $10 \mathrm{mn}$ ). We think that the instrumental drift modifies the incidence angle of the radiation on the QWPs, which results in a variation of the crossed thickness and then a spectral displacement of the fringes.

By so doing, we got a much better result on the fringe elimination by using the flat-field images, as it can be seen in our results given in Figs. 1-6, where no filtering of fringes has been applied.

The entrance slit was always oriented parallel to the tangential direction to the solar limb, a few arcsec inside the limb. To determine the distance between the limb and the slit, we allowed the solar limb to be visible on the window F1 field-of-view camera, so that an image of the limb was recorded.

To measure the position of the limb, we applied a method derived from the bisector method for obtaining the line positions (Bommier \& Rayrole 2002, Sect. 3.1). We first averaged each limb image along a direction parallel to the slit. Then, we determine the mean inflection point of the limb in the whole sequence data (Col. $\mathrm{j}$ of Table 3) and the standard deviation from the individual images (uncertainty of the same column). The noise signal is reduced by mean of a convolution of the signal with a stepwise function of width $n_{\mathrm{s}}$. We determine the position of the zero of the $d$ function defined by

$d(i)=p\left(i+n_{\mathrm{g}}\right)+p(i)-2 p\left(i+n_{\mathrm{g}} / 2\right)$,

which replaces Eq. (2) of Sect 3.1 of Bommier \& Rayrole (2002), $i$ is the pixel number, $p(i)$ is the convolved signal, and $n_{\mathrm{g}}$ must be now an even integer number. For the present data, we used $n_{\mathrm{s}}=7$ and $n_{\mathrm{g}}=6$.

The observational strategy was to interleave the limb observation $\sim 10$ min frames with flat-field records. So doing, we denoted two distinct contributions to the standard deviation given as uncertainty in Col. $\mathrm{j}$ of Table 3: (a) the standard deviation of the limb position determined as described above on individual images, during each scan; (b) the contribution of the manual correction of the telescope drift between the scans. Both effects contribute to the standard deviation. The uncertainty on the limb position is given at least by this standard deviation: seeing effects could increase this uncertainty.

We used two different values for the CCD optics magnifying factor $\gamma$ as indicated in the Col. e of Table 3 . The spectral resolution, i.e., the spectrograph bandwidth, is given by the Col. f of Table 3, in pm. The corresponding value in terms of camera pixel is given in Col. $g$ of Table 3: the magnifying factor value $\gamma=0.23$ corresponds to a sampling of 1 pixel per spectral element of resolution while the value of 0.44 corresponds to twice that with a loose of a factor of 2 in terms of flux (a factor of 2 and not 4 due to the special optical set-up of the MTR with THEMIS).

\section{Data processing technique}

We averaged all the frames along a direction parallel to the slit, to increase the polarimetric sensitivity (degrading the temporal and spatial resolution but keeping in mind that we observed "a priori" quiet regions). So doing, we obtain spectra for different Stokes parameters. Figures 1-6 show the polarization signal $X / I$ of each Stokes parameters $X=$ $Q, U$ or $V$. We plotted the intensity $I$ in dotted lines, in arbitrary units.

The data analysis method is basically described in Bommier \& Rayrole (2002).

\subsection{Fringes}

Two embedded spectral fringe patterns were present in the frames: a nearly vertical one with the same spectral variation for different slit points and an interfringe of 200 pixels, and other one more inclined with an interfringe of 50 pixels. We noticed a change of the fringe shape when using 
the different analyzer positions. So, we suppose that these fringes could be formed in the analyzer made up of two quarter-wave plates.

Assuming that there is interference between rays that have been reflected on the two faces of a given plate, and rays that have not been reflected, the interfringe is given by a unit variation of the difference of optical paths

$\frac{\varphi}{2 \pi}=\frac{2 d n}{\lambda}$

where $d$ is the plate thickness, $n$ is the index and $\lambda$ is the wavelength. This leads to

$d n=\frac{1}{2} \frac{\lambda^{2}}{\Delta \lambda}$,

where $\Delta \lambda$ is the interfringe.

We obtain a value of $d n=0.412 \mathrm{~mm}$ for the 200 pixel interfringe corresponding to the plate thickness, and a value of $d n=1.648 \mathrm{~mm}$ for the 50 pixels interfringe corresponding to the separation between the two plates. The inclination of the 50 pixel fringes could be associated with the orientation of the two plates together with respect to the optical axis.

\subsection{Line positions}

We exclusively applied the bisector method to determine the line positions, as described in Sect. 3.1 of Bommier \& Rayrole (2002). Nevertheless, we applied another data analysis strategy to correct for the difference of dispersion: by applying the Fourier interpolation method described in Bommier \& Rayrole (2002, Sect. 3.2.2), we ascribed a fixed integer position difference (identical for flat field and limb images) between two lines chosen in the field for this purpose.

\subsection{Generalized beam exchange method}

We present in Appendix A a generalization of the beam exchange technique to overcome the instrumental limitation of THEMIS polarimeter. During this observational period, the beam exchange was available only for one single Stokes parameter. Section 4 and Table 4 show that we have reached nearly the same polarization accuracy. The noise level of spectra obtained with the generalized beam exchange method (Cols. $m$ and o) are of the same order as the noise computed when using the usual method (Col. $n$ ). Higher values of the noise shown in Cols. $m$ and o correspond to a combination of six images while only four images were necessary to apply the usual beam exchange technique (Col. n).

\section{Results of observations}

The aim of this section is to discuss the results of our observations in terms of polarization measurements.

Notice that in the following, the reference system for the Stokes parameters is now defined with respect to the
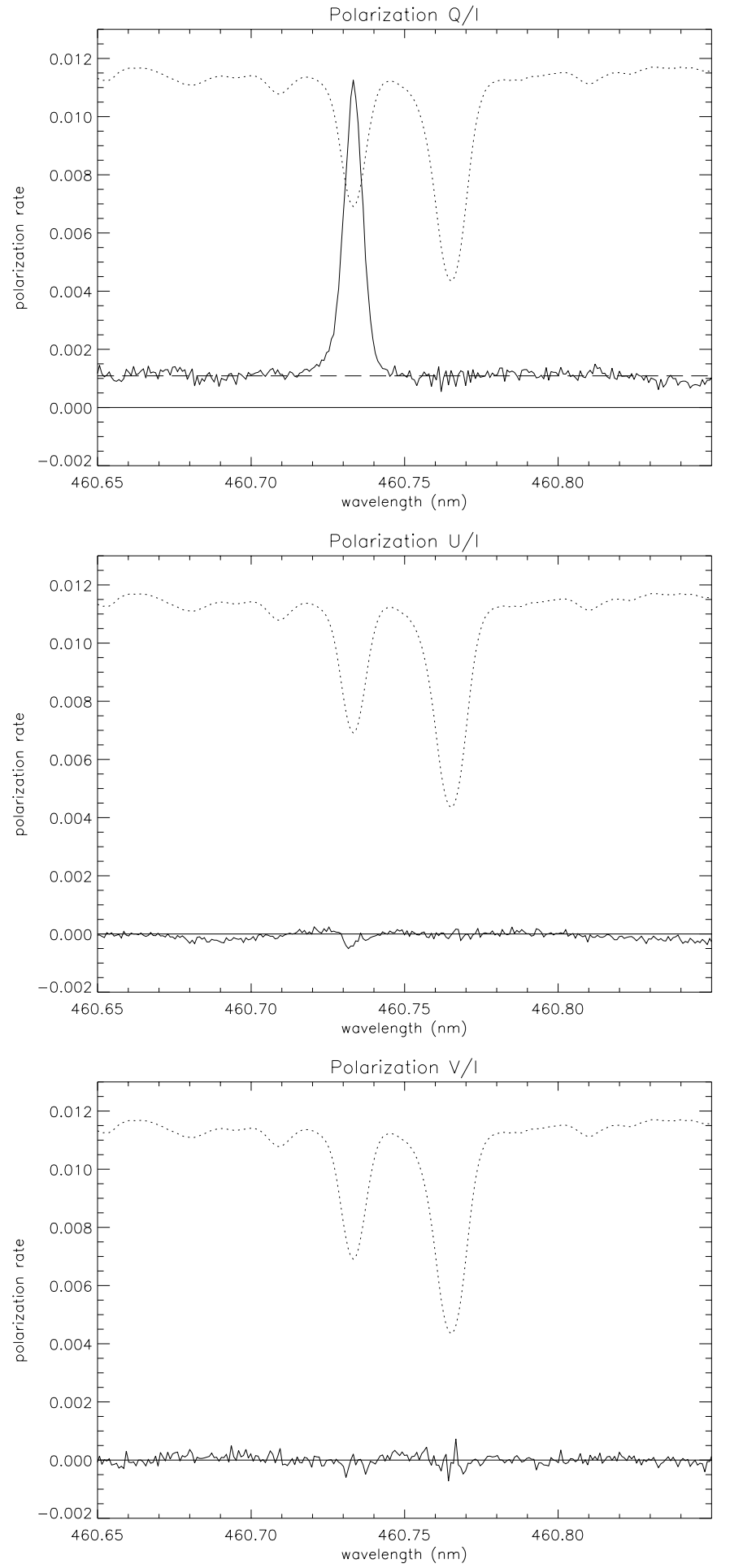

Fig. 1. Polarization of the Sr I line at $460.73 \mathrm{~nm}$, as observed on 2000 August 27 at $8 \mathrm{~h} 20-9 \mathrm{~h} 19$ UT, at $5.9 \pm 0.4$ arcsec from the solar limb. The neighboring line at $460.77 \mathrm{~nm}$ is a Fe I line.

solar limb, i.e., the $O X$ axis (the "positive $Q$ direction") is parallel to the limb direction. By comparison, in the previous Sect. 2, the $Q$ and $U$ parameters are interchanged, because in that previous section the Stokes parameters were defined with respect to the beam-splitter axes, which are $45^{\circ}$ from the spectrograph entrance slit (that has been 

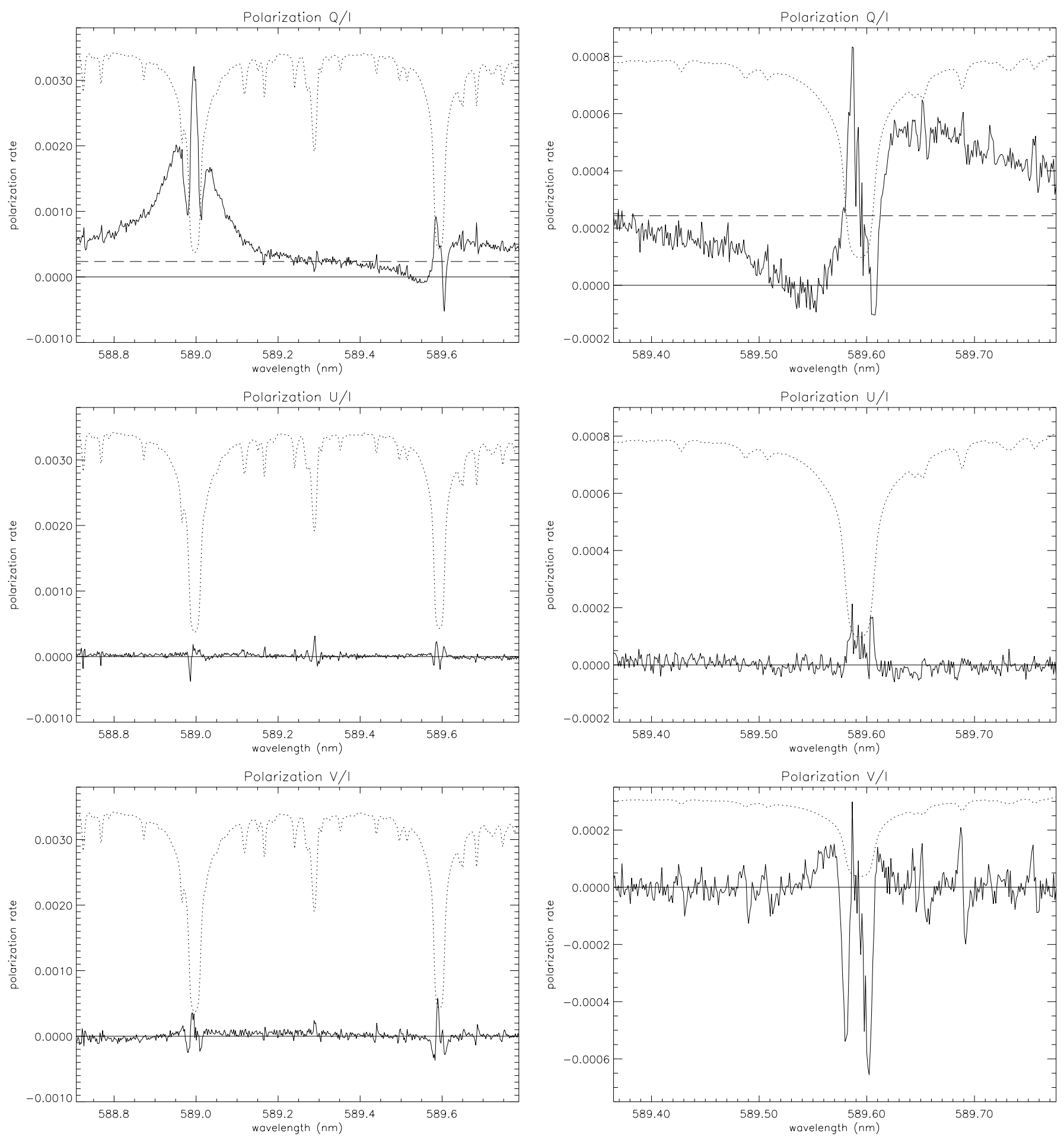

Fig. 2. Polarization of the $\mathrm{NaI}_{\mathrm{I}} \mathrm{D}_{1}$ and $\mathrm{D}_{2}$ lines at 589.6 and $589.0 \mathrm{~nm}$ respectively, as observed on 2000 August 29 at $7 \mathrm{~h} 52$ $10 \mathrm{~h} 03 \mathrm{UT}$, at $4.1 \pm 0.5$ arcsec from the solar limb. The central line at $589.3 \mathrm{~nm}$ is a $\mathrm{Ni}$ I line.

positioned parallel to the limb). The knowledge of the sign of $U$ requires however further instrumental investigation.

In the present reference system (limb reference direction), the beam exchange facility was available for the $U$ Stokes parameter only, during the 2000 campaign of THEMIS. Thus, the present results on $U / I$ have been obtained by applying the usual beam exchange technique of

Fig. 3. Polarization of the $\mathrm{Na}_{\mathrm{I}} \mathrm{D}_{1}$ line at $589.6 \mathrm{~nm}$, as observed on 2000 August 30 at $16 \mathrm{~h} 14-17 \mathrm{~h} 09 \mathrm{UT}$, at $3.8 \pm 0,6$ arcsec from the solar limb. The magnifying factor used for the camera optics is twice the one of Fig. 2.

analysis, whereas the present results on $Q / I$ and $V / I$ have been obtained by applying our generalized beam exchange technique.

No fringe filtering (Fourier or wavelets) has been applied to the results presented in the figures and in the following. 

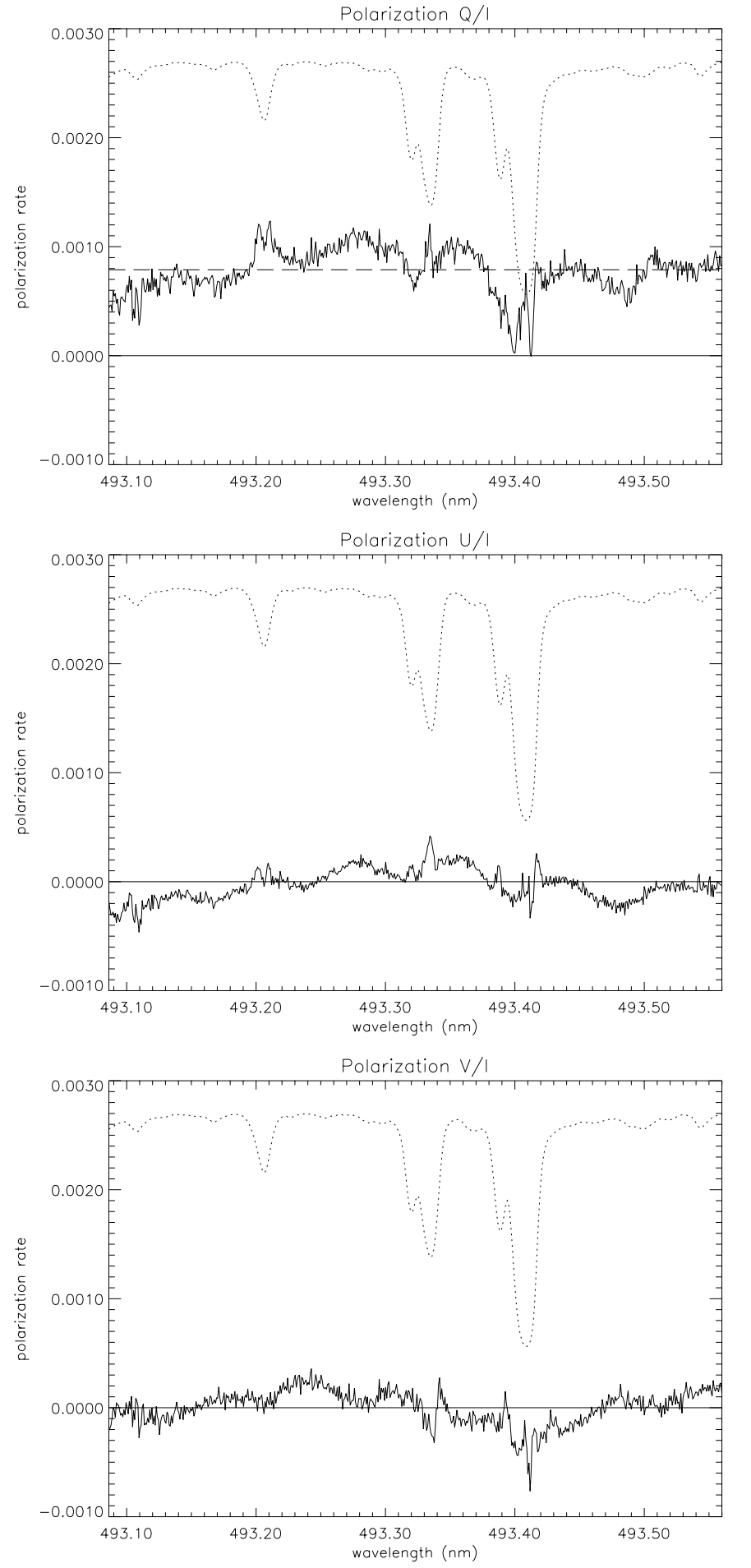

Fig. 4. Polarization of the $\mathrm{C}_{\mathrm{I}}$ line at $493.21 \mathrm{~nm}$, and of the Ba II $D_{1}$ line at $493.41 \mathrm{~nm}$, as observed on 2000 September 1 at $8 \mathrm{~h} 33-10 \mathrm{~h} 06 \mathrm{UT}$, at $4.2 \pm 0.5$ arcsec from the solar limb. The other lines are Fe I lines.

The level of continuum polarization plotted in dashed line in our figures has been derived from Eqs. (17)-(19) of Fluri \& Stenflo (1999), by applying their model 5 (which is the C model of Fontenla et al. 1993, corresponding to the average Quiet Sun), and by applying the limb darkening

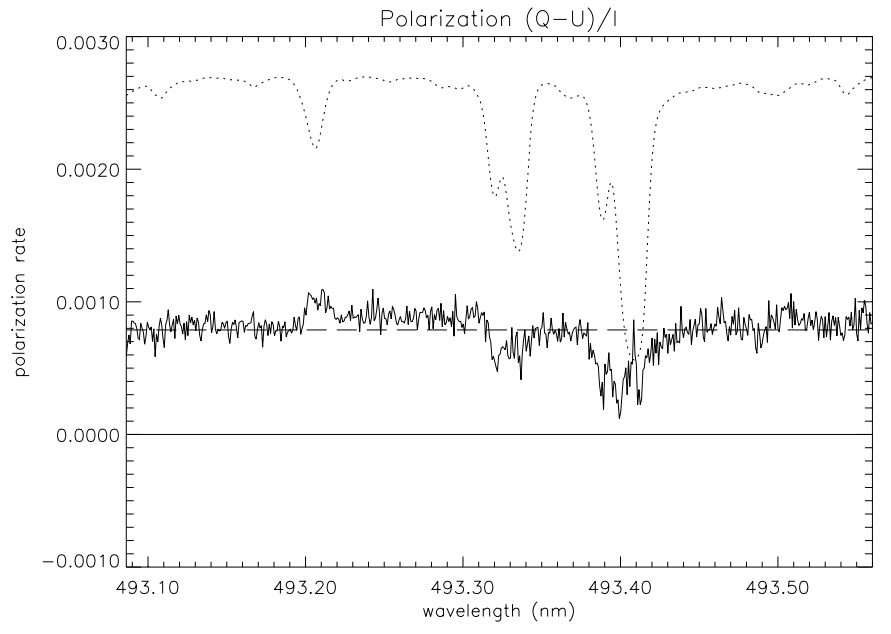

Fig. 5. Polarization of the $\mathrm{C}_{\mathrm{I}}$ line at $493.21 \mathrm{~nm}$, and of the Ba II $D_{1}$ line at $493.41 \mathrm{~nm}$, as observed on 2000 September 1 at $8 \mathrm{~h} 33-10 \mathrm{~h} 06 \mathrm{UT}$, at $4.2 \pm 0.5 \mathrm{arcsec}$ from the solar limb. The other lines are Fe I lines.

Table 5. Linear polarization of the Sr I $460.7 \mathrm{~nm}$ line.

\begin{tabular}{cccc}
\hline $\begin{array}{c}\text { Obs } \\
\#\end{array}$ & $\begin{array}{c}d_{\text {limb }} \\
\operatorname{arcsec}\end{array}$ & $\mu$ & $\begin{array}{c}{[Q / I]_{\max }} \\
\times 10^{-2}\end{array}$ \\
\hline$\# 1$ & $5.9 \pm 0.4$ & $0.110 \pm 0.004$ & 1.13 \\
$\# 2$ & $7.0 \pm 0.2$ & $0.121 \pm 0.002$ & 1.03 \\
$\# 3$ & $3.4 \pm 0.4$ & $0.084 \pm 0.005$ & 1.17 \\
\hline
\end{tabular}

$L_{4}(\mu)$ function as given by Neckel (1996), with a cubic spline interpolation in the data tables.

Observations \#1-3 were devoted to the limb polarization of the Sr I line at $460.73 \mathrm{~nm}$, at various limb distances. Results are given in Fig. 1 for Obs. \#1; similar results have been obtained for Obs. \#2-3. We set the magnifying factor of the camera optics at 0.44 to have a spectral sampling of $\sim 2$ pixels per spectral resolution element (as indicated in Table 3). No circular polarization is observed $(V / I=0)$, and the linear polarization is found to be parallel to the solar limb $(U / I=0$; the only non-zero Stokes parameter is $Q / I$ ). The maximum linear polarization observed in the Sr I line is reported in Table 5. One can see the decrease of the linear polarization with increase in the distance from the limb. The neighbor line, a Fe I line at $460.77 \mathrm{~nm}$, presents no linear polarization with respect to the level of continuum polarization given as a dashed line.

Observations \#4-11 were devoted to the limb polarization of the $\mathrm{NaI} \mathrm{D}_{1}$ and $\mathrm{D}_{2}$ lines, respectively at 589.6 and $589.0 \mathrm{~nm}$. Observations \#4-6 are reported in Fig. 2; similar results have been obtained for Obs. \#7-11. For the observations reported in Fig. 2, we set the magnifying factor of the camera optics at 0.23 to have a sampling of one pixel per spectral resolution element (as indicated in Table 3). Notice that the size of the CCD is 382 pixels along the spectral dimension and corresponds to a spectral window of $\sim 0.65 \mathrm{~nm}$ width. So, the figure is the combination of 3 spectral windows, separately observed and 

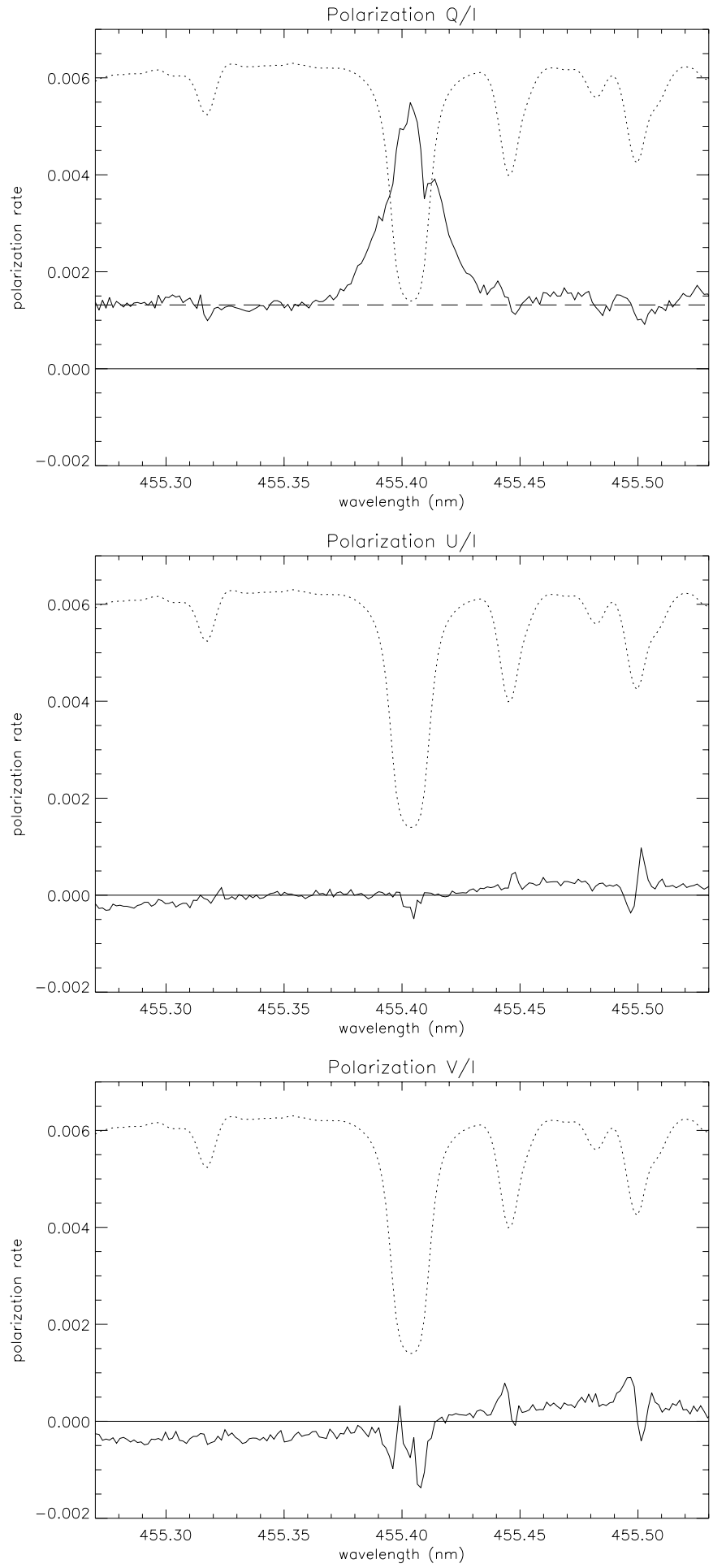

Fig. 6. Polarization of the Ba II $\mathrm{D}_{2}$ line at $455.40 \mathrm{~nm}$, as observed on 2000 September 1 at 16h09-17h15 UT, at $4.8 \pm$ 1.0 arcsec from the solar limb. The line at $455.50 \mathrm{~nm}$ is a Cr II line.

respectively centered at 589.0, 589.3 and $589.6 \mathrm{~nm}$. The absorption line in the middle one between the two $\mathrm{Na}_{\mathrm{I}} \mathrm{D}_{1}$ and $\mathrm{D}_{2}$ lines is a Ni I line. Obs. \#11 is reported in Fig. 3, that is centered on the $\mathrm{NaI}_{1}$ line; the value of magnifying factor is 0.44 , corresponding to $\sim 2$ pixels per spectral resolution element. In all these observations, as in the case of the Sr I line, no circular polarization is observed
$(V / I=0)$, and the linear polarization is found to be parallel to the solar limb $(U / I=0$; the only non-zero Stokes parameter is $Q / I)$. The shape of the linear polarization is in good agreement with the theoretical modeling of Landi Degl'Innocenti (1998). In particular, there is no global polarization in the $\mathrm{D}_{1}$ line at $589.6 \mathrm{~nm}$, when integrating over frequencies (one observes a purely antisymmetrical polarization shape, with respect to the line center) in contrast to the previous observations of Stenflo \& Keller (1997) and Stenflo et al. (2000a, 2000b), but in agreement with the polarization profile reported in the second solar spectrum atlas of Gandorfer (2000), and also with profiles observed nearer to the limb by Keller \& Sheeley (1999). Further theoretical as well as observational investigations have to be done to fully clarify this point.

As for the polarization degree, our results are in full agreement with those of Arnaud et al. (2001), who have observed the Sr I $460.7 \mathrm{~nm}$ line and the Na I D lines with THEMIS, at the same limb distance and during the same campaign, but with a different observation and reduction technique (their slit was oriented $45^{\circ}$ from the solar limb). Full agreement is obtained also with the results of Trujillo Bueno et al. (2001), who observed the Sr I $460.7 \mathrm{~nm}$ line closer to the limb, also with THEMIS during the same campaign. Their observation and reduction technique are closer to ours, and a detailed comparison of both reduction techniques has led to full agreement. Both teams used a different intrumental setting, recording both images on a single camera, whereas we used two different cameras, one camera per image.

Figure 4 presents the observations \#12-13. The figures are also the combination of two separately observed spectral windows. For these observations, the value of the selected magnifying factor was 0.44 to obtain a sampling of $\sim 2$ pixels per spectral resolution element (as indicated in Table 3). The lines are a $\mathrm{C}$ I line at $493.21 \mathrm{~nm}$, and the $\mathrm{Ba}$ II $\mathrm{D}_{1}$ line at $493.41 \mathrm{~nm}$, and $4 \mathrm{Fe}$ I lines. Moerover, there is an extra $\mathrm{Fe}$ I line that blends with the $\mathrm{Ba}$ II $\mathrm{D}_{1}$ one (see Gandorfer 2000). There is also a V I line that blends with the C I one (see Stenflo et al. 2000b; Gandorfer 2000). In this spectral range, the pertubation of the residual fringe patterns is the most important of all our observations. The line polarizations are weak and the residual fringes are clearly apparent in the figures.

Due to the presence of fringe patterns in the continuum of the polarization spectra and the absence of a polarized line in the $U / I$ spectrum, we subtracted this $U / I$ spectrum, as it was a polarization zero level, from $Q / I$. Figure 5 shows the linear polarization parallel to the solar limb. In this figure, we denote an excess of polarization of the $\mathrm{C}_{\mathrm{I}}$ line of $2-3 \times 10^{-4}$ in comparison with the continuum, in good agreement with the results of Gandorfer (2000), but lower by a factor of 2 than the results of Stenflo et al. (2000b). In Fig. 5, the depolarization in the Fe I lines is clearly apparent, in agreement also with Gandorfer (2000). Moreover, and in agreement also with Gandorfer (2000), no polarization peak in the $493.41 \mathrm{~nm}$ line appears, as observed by Stenflo et al. (2000b), that 
they attribute to the $\mathrm{BaII} \mathrm{D}_{1}$ line, which is nevertheless an unpolarizable line having $J=1 / 2 \rightarrow J^{\prime}=1 / 2$.

Figure 6 presents the result of observation \#14, devoted to the BaII $\mathrm{D}_{2}$ line at $455.40 \mathrm{~nm}$. Although the seeing was the worst on that day, it can be seen that the polarization profile shows the same structure in the wings, as previously observed by Stenflo \& Keller (1997), that they have attributed to the hyperfine structure of the odd isotopes, whereas the central polarization peak is due to the even isotopes. However, we have observed a linear polarization rate of $5.5 \times 10^{-3}$ in the line center, that is a factor of 2 lower than that observed by Stenflo \& Keller (1997) at the same limb distance.

\subsection{Eventual 11-year cyclic variation}

We point out that the observed polarization of the Sr I $460.7 \mathrm{~nm}$ line as shown in Fig. 1, together with the center-to-limb polarization decrease reported in Table 5, are smaller than the ones observed in June and November 1994 by Stenflo et al. (1997) by a factor of 1.5. Nevertheless, results are in better agreement with earlier results (though taken farther from the limb) when comparing May-June 1978 data (Stenflo et al. 1980) with a rather low spectral resolution, and October 1978 and April 1979 data (Stenflo 1983) with a better spectral resolution (the Kitt Peak Mc Math telescope FTS was used). Moreover, as already stated, our results are in full agreement with those obtained by Arnaud et al. (2001) and Trujillo Bueno et al. (2001), with the same instrument and during the same campaign. This suggests cyclic variations of the limb polarization that would follow the 11-year solar cycle because, with respect to this cycle, the 78 and 79 data are in phase with the present 2000 ones, whereas the 94 data are in phase opposition.

The observed polarization in the central peak of the $\mathrm{Na}$ I $\mathrm{D}_{2}$ line at $589.0 \mathrm{~nm}$ is of the order of $0.3 \%$ at $4 \operatorname{arcsec}$ from the solar limb (see Figs. 2-3). In April 1995, Stenflo \& Keller (1997) and Stenflo et al. (2000b) observed 0.5\% at 5 arcsec from the limb $(\mu=0.1)$, and, in March 1998, Stenflo et al. (2000a) observed $0.4 \%$ at 5 arcsec from the solar limb. In 1999-2000, Gandorfer (2000) got 0.3\% at 5 arcsec from the solar limb, in agreement with our 2000 observations. This behavior suggests also an 11-year cyclic decrease of the limb polarization.

It has already been stressed that the polarization observed in the wings of the $\mathrm{Na}$ I $\mathrm{D}_{2}$ line is lower than the one obtained by Gandorfer (2000). Nevertheless, besides considering the hypothesis of an eventual 11-year cyclic variation of the limb polarization, it is highly desirable to achieve some test concerning an instrumental depolarization effect of the entrance BK7 window of THEMIS tube telescope before interpreting any results.

As stated before, the $\mathrm{C}_{\mathrm{I}}$ line shows an excess of polarization of $2-3 \times 10^{-4}$ in comparison with the continuum (see Fig. 5), in good agreement with the results obtained by Gandorfer (2000) with 1999-2000 data, but lower than the results obtained by Stenflo et al. (2000b) by a factor of 2 with 1995 and 1996 data. Similarly, the linear polarization rate observed in the BaII $\mathrm{D}_{2}$ line, is a factor of 2 lower than the one observed in April 1995 by Stenflo \& Keller (1997). These features are also in agreement with an 11-year cyclic decrease of the limb polarization.

\section{Conclusion}

The generalized beam exchange technique, associated with the observational strategy presented in this paper, is a powerful technique for the measurement of weak polarization signals with THEMIS during the 2000 observational period. Despite the avaibility of beam exchange for only a single Stokes parameter, we reached the $10^{-4}$ accuracy level of polarization measurements for all the Stokes parameters.

Further observations and investigations have to be performed in order to confirm the obtained results. We have not observed global positive polarization (i.e., when integrated over frequencies), in the $\mathrm{D}_{1}$ lines, contrarily to some other observations, but in agreement with other ones and in accordance with theoretical expectations and models. Notice that we have always observed a linear polarization that is antisymmetrical with respect to the line center for Na I $D_{1}$ line.

We have generally measured polarization rates in agreement with those given in the second solar spectrum atlas of Gandorfer (2000), observed in 1999-2000. Notice that our polarization rates are systematically smaller than previous results obtained in the 94-96 years in phase opposition with respect to the 11-year solar cycle. This suggests that the limb polarization could have an 11-year cyclic variation. This hypothesis has to be investigated by further observations but it is highly desirable to investigate also the possibility of an eventual instrumental depolarization effect on THEMIS.

Acknowledgements. The authors are indebted to all the THEMIS technical team, in particular to O. Grassin for his remarkable work on the user interface and his assistance during the observations. They are also indebted to J. Rayrole, J. L. Leroy and E. Landi Degl'Innocenti for fruitful discussions, and to the referee for helpful criticisms.

\section{Appendix A: Generalized beam exchange}

The aim of this section is to present a generalization of the beam exchange method (Donati et al. 1990; Bianda et al. 1998) to compensante for the incomplete beam exchange of all Stokes parameters in the situation encountered at THEMIS during the 2000 observational period.

\section{A.1. Usual beam exchange}

Let us first recall the usual method and formula for treating beam exchange data.

Considering $X$ as the observed Stokes parameter, we obtain simultaneously two beams from the beam-splitter 
of the analyzer: one of the two beams (beam \#1) gives the signal $I+X$, and the other beam (beam \#2) gives the opposite Stokes parameter, i.e., the signal $I-X$ (where $I$ is the intensity).

In the following record, the two beams are "exchanged" when moving the analyzer quarter wave plates positions to obtain the $I-X$ signal from beam $\# 1$ and the $I+X$ signal from beam \#2.

We adopt the notation $I X$ ("inverse of $X$ ") to qualify the beam exchange of the Stokes parameter $X$. So, after the exchange, beam \#1 gives the signal $I+I X$ while beam $\# 2$ gives $I-I X$ :

$I X=-X$.

One usually derives the polarization $X / I$ by performing the following operation (Donati et al. 1990; Bianda et al. 1998):

$\frac{X}{I}=\frac{1}{4}\left[\frac{(I+X)(I-I X)}{(I-X)(I+I X)}-1\right]$.

Notice that each term in parentheses corresponds to the four images obtained before and after the beam exchange: $(I+X)$ is the signal coming from the beam \#1 before the beam exchange while $(I+I X)$ is the signal obtained after the beam exchange, $(I-X)$ is the signal coming from the beam \#2 before the beam exchange while the $(I-I X)$ is the signal obtained after the beam exchange. This formula remains valid only if the polarization $X / I$ is of the order of few \%, i.e., a polarization accuracy lower than $10^{-4}$ (as indicated in Bianda et al. 1998, see higher order corrections).

An advantage of this method is to overcome the lack of knowledge concerning the electronic gain of the CCD cameras when dividing the two exchanged images recorded by the same detector. In that case, the flat-field is theoretically unnecessary and the computation can be performed with current images (after dark subtraction).

We present another method to perform the same operation. Due to the fact that $X / I$ (and, obviously, $I X / I$ ) are small (few \%), the previous formula becomes:

$$
\frac{X}{I}=\frac{1}{2}\left[\frac{X}{I}-\frac{I X}{I}\right] .
$$

We found two different ways to apply the formula:

(a) We can subtract frames recorded at the same time from two different cameras, and subtract the results. $X / I$ is obtained when subtracting $I-X$ from $I+X$ (and dividing by their sum), following the method described in Bommier \& Rayrole (2002). IX/I is analogously obtained by subtracting $I-I X$ from $I+I X$, leading to the final polarization $X / I$. This treatment can be expressed as:

$$
\begin{aligned}
\frac{X}{I}= & \frac{1}{2}\left[\frac{(I+X)-(I-X)}{(I+X)+(I-X)}\right. \\
& \left.-\frac{(I+I X)-(I-I X)}{(I+I X)+(I-I X)}\right] .
\end{aligned}
$$

(b) We can subtract frames taken at different time on the same camera, and subtract the results. Then the treatment can be written as:

$$
\begin{aligned}
\frac{X}{I}= & \frac{1}{2}\left[\frac{(I+X)-(I+I X)}{(I+X)+(I+I X)}\right. \\
& \left.-\frac{(I-X)-(I-I X)}{(I-X)+(I-I X)}\right] .
\end{aligned}
$$

As expected, both methods give comparable results. It appears clearly when using the second combination (b) that the beam exchange technique averages the signals obtained from different cameras: differences between cameras or channels such as focus or elecronic gain fluctuations are averaged rather than subtracted, and so far, the polarization analysis is not further disturbed by them. Notice that the eventual scattered or stray light is suppressed, together with the dark current fluctuations slower than the integration time when subtracting the two different signal obtained at different times on the same detector. The beam exchange technique is also free of temporal variations of the atmosphere or instrumental transmission. All these characteristics make the beam exchange technique (even on two different cameras) much more efficient than the usual polarization analysis. We reach the $10^{-4}$ level (see Table 4) while the usual polarization analyses as the method of Bommier \& Rayrole 2002) are limited to the $10^{-3}$ polarization level in the $460 \mathrm{~nm}$ wavelength range.

As previously remarked, it appears that the flat-field correction would not be necessary, with regard to the equivalence between Eqs. (A.2) and (A.3), only if the fringe problem is not considered. Different positions of the crystalline quarter-wave plates to analyze the Stokes parameters $X$ and $I X$ create different fringe patterns which cannot be eliminated when using the beam exchange technique. Fortunately, this inconvenience can be corrected by using the flat-field images and assuming that they are unpolarized. The same combination of the flat-field images provides the (first) polarization zero level, that contains only the fringes, which can then be subtracted from the same combination of limb images. Alternatively, each limb image has to be divided by the corresponding flat-field image, when Eq. (A.2) is applied. The correction vector method of Bommier \& Rayrole (2002), when applied to individual 2-image combinations, leads to the same result.

\section{A.2. Generalization of the beam exchange}

Let us assume now that the Stokes parameter $X$ is observed (the two beams provide $I+X$ and $I-X$ respectively). In the instrumental configuration, only one beam exchange is available for a different Stokes parameter, denoted $Y$. So, we get the four signals $I+Y, I-Y, I+I Y$, $I-I Y$. The definition of $I Y$ is analogous to the one of $I X$ in Eq. (A.1).

Remarking that the dark current fluctuations are highly reduced by combining two images taken at different times on the same camera, the idea is to achieve 
such a subtraction between $X$ and $Y$, and then to add the "true" $Y$ derived from the beam exchange analysis described above. Thus, the final polarization $X / I$ can be retrieved by combining the six images $I+X, I-X, I+Y$, $I-Y, I+I Y, I-I Y$, following

$$
\frac{X}{I}=\frac{X}{I}-\frac{Y}{I}+\frac{1}{2}\left[\frac{Y}{I}-\frac{I Y}{I}\right]
$$

This formula means that the $I+Y$ image is subtracted from the $I+X$ image, following for instance the method of Bommier \& Rayrole (2002). In this method, we subtract the two signals obtained at the same time but on two different cameras. Now, we subtract $I+X$ and $I+Y$ at different times but on the same camera. So doing, $I+I Y$ is subtracted in the same way from $I+Y$, and then Eq. (A.6) is applied. The same analysis is performed in the other channel, by combining $I-X, I-Y, I-I Y$, and the two results are then averaged (the second result is the opposite). This can be written as

$$
\begin{aligned}
\frac{X}{I}= & {\left[\frac{(I+X)-(I+Y)}{(I+X)+(I+Y)}+\frac{1}{2} \frac{(I+Y)-(I+I Y)}{(I+Y)+(I+I Y)}\right](\text { A.7 }) } \\
& -\left[\frac{(I-X)-(I-Y)}{(I-X)+(I-Y)}+\frac{1}{2} \frac{(I-Y)-(I-I Y)}{(I-Y)+(I-I Y)}\right] .
\end{aligned}
$$

Indeed, when $I+Y$ is subtracted from $I+X$, and when the difference is divided by the sum which is $2 I+X+Y, X+Y$ is neglected before $2 I$ in the denominator. $X / I$ and $Y / I$ being of the order of a few $\%$, such an analysis is valid only up to the $10^{-4}$ level in polarization accuracy. This method separately combines the images taken at different times on the same camera, and then averages the channels.

Alternatively, Eq. (A.6) can be rewritten as

$$
\frac{X}{I}=\frac{X}{I}-\frac{1}{2}\left[\frac{Y}{I}+\frac{I Y}{I}\right]
$$

which suggests another way of performing the operation: each couple of images taken at the same time on the two different cameras is treated following the method of Bommier \& Rayrole (2002). One thus obtain separately $X / I, Y / I$, and $I Y / I$, and Eq. (A.8) is then applied. This can be written as

$$
\begin{aligned}
\frac{X}{I}= & \frac{(I+X)-(I-X)}{(I+X)+(I-X)} \\
& -\frac{1}{2}\left[\frac{(I+Y)-(I-Y)}{(I+Y)+(I-Y)}+\frac{(I+I Y)-(I-I Y)}{(I+I Y)+(I-I Y)}\right] .
\end{aligned}
$$

The physical meaning of Eq. (A.8) is that, while $[Y / I-I Y / I] / 2$ provides the "true" $Y / I$ (corrected for the dark current fluctuations), $Y / I+I Y / I$ provides the (second) polarization zero level (that includes the dark current fluctuations). The first polarization zero level is given by the correction vector of Bommier \& Rayrole (2002), or by the same combination applied to the flat-field images, that are assumed to be unpolarized.

We have performed these two ways of image combination with our 2000 data, and found no noticeable difference in the derived results.
For the generalized beam exchange, the analogous formula of Eq. (A.2), that can be directly applied to the images, is then:

$$
\frac{X}{I}=\frac{1}{2}\left[\frac{(I+X)}{(I-X)} \sqrt{\frac{(I-Y)(I-I Y)}{(I+Y)(I+I Y)}}-1\right] .
$$

The present "generalized beam exchange" technique is in fact the same as the so-called "spatio-temporal modulation method" discussed by Trujillo Bueno et al. (2001).

\section{References}

Arnaud, J., Faurobert, M., Vigneau, J., \& Paletou, F. 2001, in Advanced Solar Polarimetry: Theory, Observations and Instrumentation. Proceedings of the 20th International Sacramento Peak Summer Workshop, ed. M. Sigwarth, ASP Conf. Ser., 236, 151

Bianda, M., Solanki, S. K., \& Stenflo, J. O. 1998, A\&A, 331, 760

Bommier, V., \& Rayrole, J. 2002, A\&A, 381, 227

Donati, J. F., Semel, M., Rees, D. E., Taylor, K., \& Robinson, D. 1990, A\&A, 232, L1

Faurobert, M. 1987, A\&A, 178, 269

Faurobert, M. 1988, A\&A, 194, 268

Faurobert-Scholl, M. 1993, A\&A, 268, 765

Faurobert-Scholl, M., Feautrier, N., Machefert, F., Petrovay, K., \& Spielfiedel, A. 1995, A\&A, 298, 289

Fluri, D. M., \& Stenflo, J. O. 1999, A\&A, 341, 902

Fontenla, J. M., Avrett, E. H., \& Loeser, R. 1993, ApJ, 406, 319

Gandorfer, A. 2000, The Second Solar Spectrum, a high spectral resolution polarimetric survey of scattering polarization at the solar limb in graphical representation. Hochschulverlag AG an der ETH Zürich

Keller, C. U., \& Sheeley, N. R. Jr 1999, Proceedings of the 2nd Solar Polarization Workshop, ed. K. N. Nagendra, \& J. O. Stenflo, ASSL Ser. 243 (Kluwer, Dordrecht), 17

Landi Degl'Innocenti, E. 1995, SPW1 Proc., St Petersburg (also Sol. Phys. 164, 21)

Landi Degl'Innocenti, E. 1998, Nature, 392, 256

López Ariste, A., Rayrole, J., \& Semel, M. 2000, A\&AS, 142, 137

Neckel, H. 1996, Sol. Phys., 167, 9

Stenflo, J. O., Baur, T. G., \& Elmore, D. F. 1980, A\&A, 84, 60

Stenflo, J. O., Twerenbold, D., Harvey, J. W., \& Brault, J. W. 1983, A\&AS, 54, 505

Stenflo, J. O., \& Keller, C. U. 1997, A\&A, 321, 927

Stenflo, J. O., Bianda, M., Keller, C. U., \& Solanki, S. K. 1997, A\&A, 322, 985

Stenflo, J. O., Gandorfer, A., \& Keller, C. U. 2000a, A\&A, 355, 781

Stenflo, J. O., Keller, C. U., \& Gandorfer, A. 2000b, A\&A, 355, 789

Trujillo Bueno, J., Collados, M., Paletou, F., \& Molodij, G. 2001, in Advanced Solar Polarimetry: Theory, Observations and Instrumentation. Proceedings of the 20th International Sacramento Peak Summer Workshop, ed. M. Sigwarth, ASP Conf. Ser., 236, 141 